\title{
Study on the Thermo-compression Deformability Rule of Aluminum Alloy Ring under Partial Restriction
}

\author{
Shi Wen-yong ${ }^{* 1,2}$, Ma Yan ${ }^{1}$, Yang Chun-mei ${ }^{1}$ and Li Feng ${ }^{3}$ \\ ${ }^{1}$ College of Mechanical and Electrical Engineering, Northeast Forestry University, \\ Harbin, China \\ ${ }^{2}$ Graduate School, Harbin University of Science and Technology, Harbin, China \\ ${ }^{3}$ College of Material Science and Engineering, Harbin University of Science and \\ Technology, Harbin, China \\ shiwenyong@hrbust.edu.cn
}

\begin{abstract}
For the sake of revealing the influence of partial restriction conditions on the thermocompression deformability of the aluminum alloy ring, this paper adopts the integrated method of numerical modeling and technology to make deep research by taking 7075 aluminum alloy as an example. It turns out that: after being exerted partial restriction, the branch surface will be at the two outside ends during the thermo-compression deformability. Compared with the situation without restriction, after being exerted partial restriction with the protruding model pressure of $8 \mathrm{~mm}$, the previous increasing of the internal and external diameter of the ring is changed to the deformability model that the internal diameter decreases and the external diameter increases. Besides, both the internal and external outlines are in the shape of evagination and drum, with the maximum increasing thickness of the side wall from $24.6 \%$ to $51.2 \%$. Therefore, restriction imposing can effectively improve the thermo-compression liquidity of the ring, which provides theory basis for the technology preform and deformability liquidity control during the precise formability of the complex components.
\end{abstract}

Keywords: aluminum alloy; ring; thermo-compression; partial restriction; deformability rule

\section{Introduction}

Cylinder or ring upsetting is one of the necessary steps for preform process of complex component formability. In order to gain needed preform shape, repeated upsetting towards the initial blank should be carried out, so as to simplify the processing procedure and reduce the cost $[1,2]$. The process condition change is to the benefit of improving the dynamic status and deformability feature of the blank [3-5]. The research results indicate that: imposing hydrostatic pressure on the outer of the ring through polytef can have an effect on the crack generation form during the compression deformability process of composite material under different conditions [6]. That hydrostatic pressure is imposed on the two sides of the ring can make it remain three-dimension pressure stress status, meanwhile, it can obviously improve the capacity of plastic deformability when compressing 7075 aluminum alloy ring [7].

In the range of $100 \mathrm{MPa}$, with the increasing of hydrostatic pressure, the limit of the testing piece increases compared to the amount of compression [8]. In addition, that hydrostatic pressure is imposed on the pressed ring can change the compression deformability feature of 
the ring [9]. This paper makes systematic research towards the compression deformability process of aluminum alloy ring under the inhomogeneous hydrostatic pressure. It turns out that: by doing so, the distribution of average stress contour can be obviously changed, namely, distribution of average stress gradient [10]. Meanwhile, inhomogeneous hydrostatic pressure gives rise to the changing of the stress field inside the testing piece, which brings obvious change for the compression deformability of the ring and metal flow direction [11].

From what has been mentioned above, most current researches are limited to the overall change of ring loading condition. The partial restriction should be imposed if the preform of special geometrical shape is needed. However, so few related researches are reported. Therefore, taking 7075 aluminum alloy ring as example, the paper makes deep research towards the compression deformability rule of the ring under the restriction imposed on the middle part of the outside.

\section{Finite Element Model and Approach}

\subsection{Finite Element Model}

Due to the complexity of three-dimensional problem, how to reasonably simplify the model and how to make it maximally close to the actual computation, become the keys of solving the problem of numerical modeling. In view of actual ring and constrained axial symmetry while reducing the quantity of unit division and improving the computation speed, this paper takes $1 / 2$ of the testing piece section as the simulation research object and adopts DEFORMTM-2D to simulate the compression process. Moreover, it adopts four hybrids and tetrahedron to make gridding division towards the blank. The finite element model is shown in Figure 1.

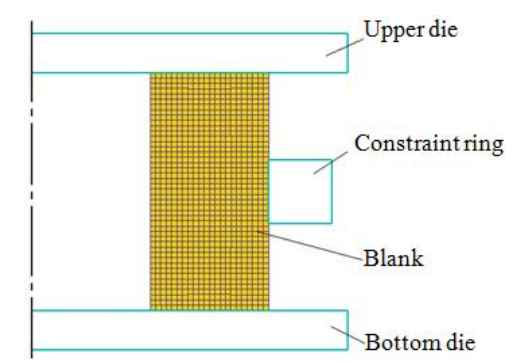

Figure 1. Finite element model

Repeat division is carried out during the simulation process in order to ensure the accuracy and convergence of computation. The blank is an ideal material of rigid plastic model. During the simulation process, the model and deformability situation of restraint ring are not taken into account. Set the attribute to rigid one.

\subsection{Research Approach}

The adopted blank is 7075 aluminum alloy ring-form blank with the external diameter of $60 \mathrm{~mm}$, internal diameter of $30 \mathrm{~mm}$ and height of $30 \mathrm{~mm}$. And the restraint ring is a stainless steel ring, which is set in the middle of the ring outside, with the external diameter of $76 \mathrm{~mm}$, internal diameter of $60 \mathrm{~mm}$ and height of $8 \mathrm{~mm}$. The formability speed is $1 \mathrm{~mm} / \mathrm{s}$. The temperature of both the testing piece and model is $430^{\circ} \mathrm{C}$. This paper adopts the iteration method of Newton-Raphson to make simulation and seek for the solution. The condition of friction boundary between model and blank is imposed based on the friction model of shear 
factor. The friction factor is measured to be 0.3 through the thermo-compression testing of the ring.

\section{Discussion and Analysis}

\subsection{Mechanical Analysis of Formability Process}

Figure 2 shows the equivalent stress distribution comparison during compression deformability process with different rolling deduction under the condition of restriction and deformability.

As shown in Figure 2a, the maximum equivalent stress of initial formability stage lies in the contact part between the inner bore end of restraint ring and the external ring, and also the inner bore ends of the ring. Only if all of these parts meet the plastic yielding conditions can they be deformed. However, the equivalent stress which is not so close to the restraint ring is weak so that it is not deformed. With the increasing of the rolling reduction shown in Figure $2 \mathrm{~b}$, the equivalent stress of the ring presents increasing trend. At the time, the maximum equivalent stress lies in the inner bore end of restraint ring and the part of external ring. The equivalent stress of the internal ring edge and the corresponding wall goes up. Except for the part of the internal restraint ring, the other parts of the ring meet the plastic yielding condition so that they are deformed. During the compression deformability process of the last formability phase, the distribution of equivalent stress is the same as before which is shown in Figure 2c. However, as the stress in the middle part of the ring increases obviously, the area coverage that does not suffer plastic deformability remarkably decreases.

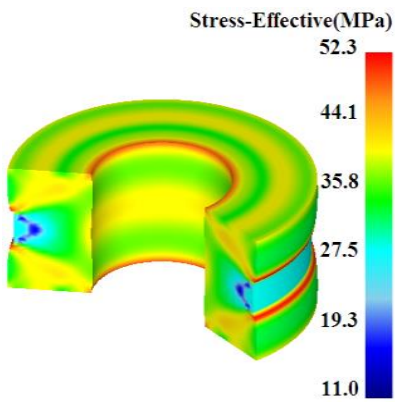

a) $3 \mathrm{~mm}$

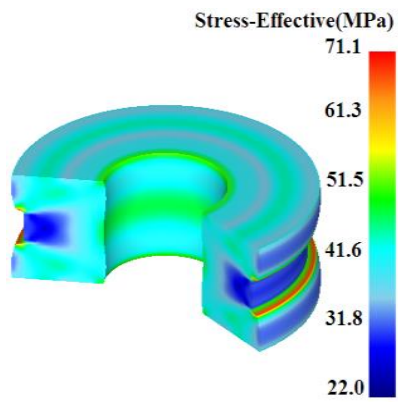

b) $6 \mathrm{~mm}$

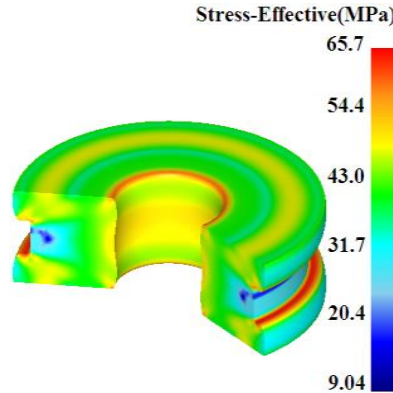

c) $8 \mathrm{~mm}$

Figure 2. Comparison of the distribution of equivalent stress under different forming stage

From the above analysis, it can be seen that, during compression deformability, the ends where the unrestraint ring and the punch-die contact together is generally the rigid area, while, after the restriction is imposed on the middle part of the ring outside, it not only changes the deformability feature of the ring, but also changes the stress state.

\subsection{Analysis of Deformability Feature}

Figure 3 shows the comparison of equivalent stress distribution during ring compression with restrictions under different rolling reduction.

As shown in Figure 3a, during the compression deformability after restrictions are carried out, except for the restraint parts, the equivalent strain of the ring outside changes a little, but it shows the change of radial outflow trend. With the increasing of rolling reduction, the size of the ring along the loading direction becomes small obviously. In Figure 3b, due to the 
effect of restriction, the external surface of the ring presents the deformability feature of upper and lower scoliosis, middle concaving, and the distribution rule of the equivalent strain value is basically in accord with the former one, and the value increases by twice. Therefore, with the increasing of rolling reduction, the deformability quantity of each part on the ring increases remarkably. The equivalent strain distribution of the last phase of formability is shown in Figure 3c. Except for the restraint parts, the external surface of the ring shows a large quantity of deformability flow along the radial direction. Besides, the two ends of the ring inner bore generates slight protruding deformability trend.

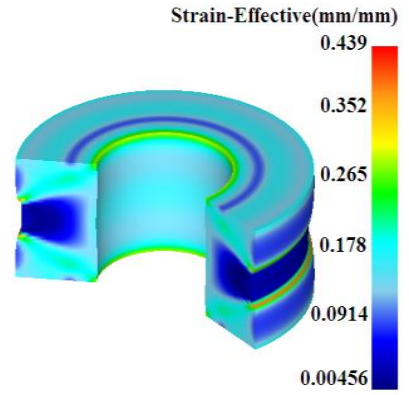

a) $3 \mathrm{~mm}$

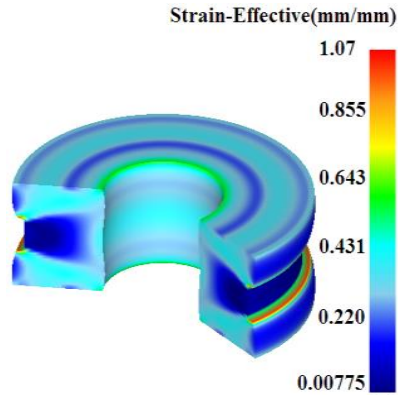

b) $6 \mathrm{~mm}$

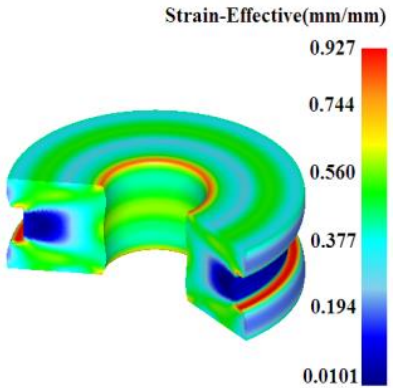

c) $8 \mathrm{~mm}$

\section{Figure 3. Comparison of the distribution of equivalent}

From what has been mentioned above, the external restriction can effectively improve the compression deformability feature. Compared to the unrestraint conditions, after the ring is imposed with compression and deformability, the deformability feature of internal and external surface will change greatly.

\subsection{Research on Metal Flow Behavior}

Except for special conditions, the flow interface will be generated during the process of compression deformability. As for the ring with restrictions, such branch surface still exists. Figure 4 shows the comparison of velocity field distribution when the restraint ring is imposed with compression deformability under different rolling reduction.

As shown in Figure 4, when the restraint ring is imposed with compression deformability, the metal flow interface which is close to the outside angle shows arc distribution, among which, the velocity of the metal on the ring ends is the largest and decreases from up to down gradually. As shown in Figure 4a, during the initial stage of the formability, the external face of the ring ends shows outflow trend along the radial direction, and the internal and middle parts are opposite. As shown in Figure 4b, with the increasing of rolling reduction, the ring ends show inside and outside branch trend along the radial direction. Therefore, the ends show the trend of evagination and double drum. In Figure 4c, during the last phase of formability, with the continuous increasing of rolling reduction, the outflow quantity of the outside ends of the ring increases and then it presents pulley-shaped change.

\subsection{Streamline Distribution Feature}

Metal streamline distribution has a direct effect on the overall performance of the components and also it can reflect the circulating rule of the metal. If the streamline distribution is uneven or has some defects, the component performance will be so difficult to meet the requirements. Therefore, analyzing the metal streamline plays an important guiding 
role in the actual production. The streamline distribution comparison of the ring compression and formability with restrictions is shown in Figure 5.

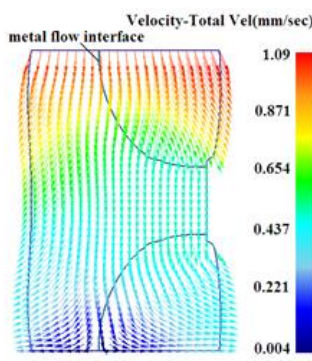

a) $3 \mathrm{~mm}$

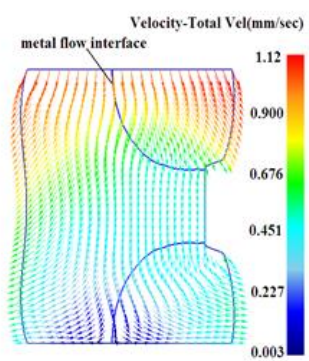

b) $6 \mathrm{~mm}$

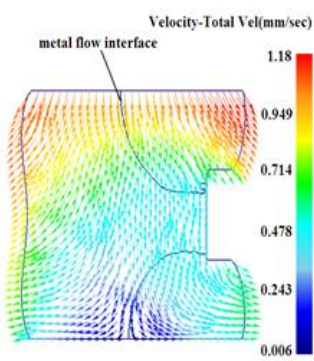

c) $8 \mathrm{~mm}$

Figure 4. Comparison of the distribution of velocity field under different forming stage

As shown in Figure 5a, during the initial period of formability, the compression deformability quantity of the ring is small, and the streamline interval seldom changes, which presents the same distribution with the shape of geometrical outline. As shown in Figure 5b, during the medium term of formability, when the rolling reduction of the upper model is $6 \mathrm{~mm}$, with the evagination trend change of the two outside ends of the ring, the streamline presents curved shape distribution and it is even and reasonable. As shown in Figure 5c, when the rolling reduction is $8 \mathrm{~mm}$, the difference of streamline distribution is great.

Due to the over large quantity of compression deformability, the distance between the upper streamline and lower streamline gets longer, especially the angle (the contacted part between the outside and restraint ring) of streamline curve degree is over large, and partial streamline shows interrupted distribution on the restraint part. This part generates cracks as it is eroded when the streamline end is exposed to the outside during usage. Therefore, although the restriction can improve the distribution of the streamline, the amount of compression should not be too large.

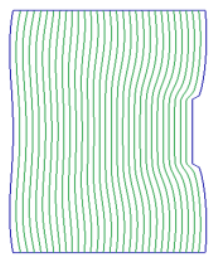

a) $3 \mathrm{~mm}$

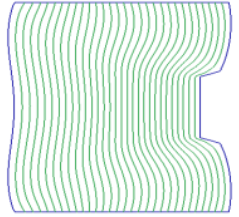

h) $6 \mathrm{~mm}$

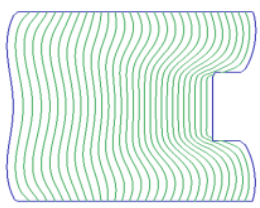

r) $8 \mathrm{~mm}$

Figure 5. Comparison of the distribution of streamline under different forming stage

\subsection{Shape Change Rule}

As the transformability difference of each part is great during the process of compression deformability, the internal and external diameter of the ring changes. In order to make further analysis, select 9 points with the same interval on the surface. The comparison of the wall thickness change of the corresponding section and the diameter with the restriction and without restriction is shown in Figure 6 and Figure 7.

As shown in Figure 6a, during the compression deformability process of the ring, the size of the internal and external diameter presents increasing trend, with the points of maximum at 
the inside and outside of the middle part respectively. When the rolling reduction value of the protruding mould reaches $8 \mathrm{~mm}$, the internal and external diameter of the corresponding part will increase by $4.2 \%$ and $14.4 \%$ respectively after the compression deformability.

With the restriction, the change trend of the diameters is obviously different from each other. As shown in Figure 6b, due to the effect of outside restriction, the material of the inner bore will deform along the radial inflow after compression so that the size of the internal diameter decreases, while the size of the outside ends obviously increases. When the rolling reduction value is $8 \mathrm{~mm}$, the maximum shrinkage of the internal diameter is $-24.7 \%$ and the maximum of the external diameter increasing is $13.7 \%$.

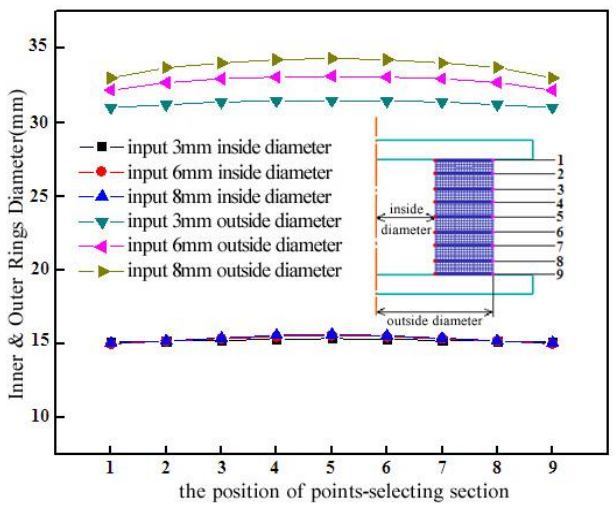

a) Nonrestraint

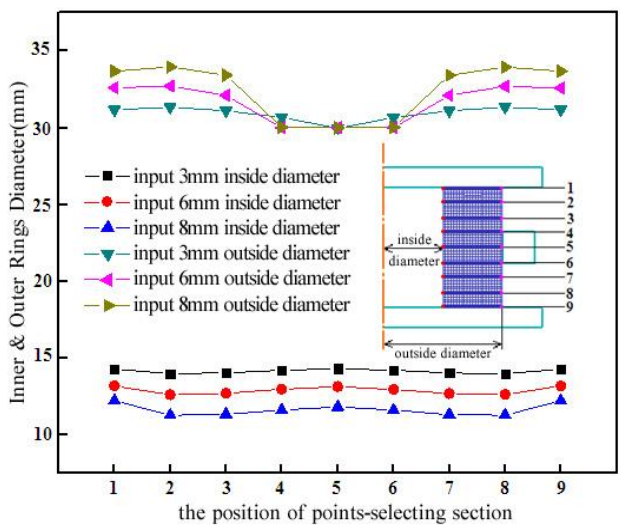

b) Constrained

Figure 6. The comparison of ring diameter change under conditions of nonrestraint and constraint

As the internal and external diameter changes during compression, the thickness of the corresponding parts will change, which is shown in Figure 7.

As shown in Figure 7a, during the process of compression deformability of unrestraint ring, with the increasing of rolling deduction, the wall thickness value of different heights presents increasing trend. When the rolling reduction value is $8 \mathrm{~mm}$, the maximum increasing value of the wall thickness is $24.6 \%$ and the point lies in the middle part of the height direction of the ring. After restriction is being imposed, the distribution of the wall thickness goes through changes.

As shown in Figure $7 \mathrm{~b}$, when the rolling reduction value is $8 \mathrm{~mm}$, the maximum wall thickness value lies on the part close to the upper and lower ends and the highest thickness 
increasing rate can reach $51.2 \%$. From the distribution of the values, although the middle part is restraint, the incrassation change takes place, with the value of $21.2 \%$. Therefore, the radial inflow quantity of the material of this part goes up with the increasing of the rolling reduction.

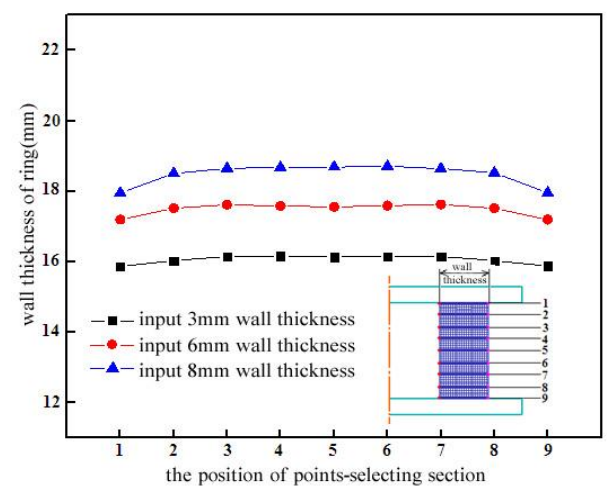

a) Nonrestraint

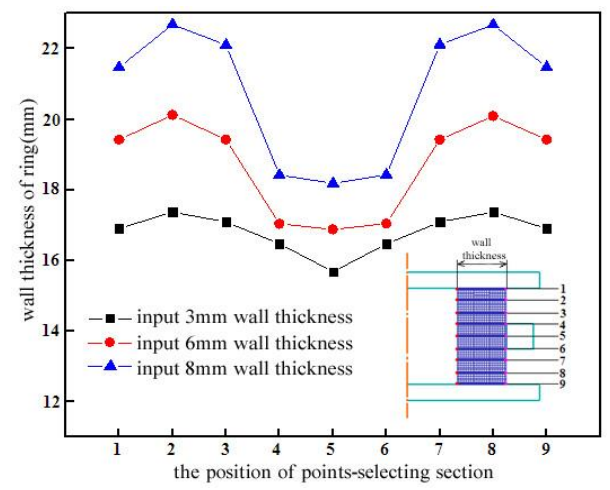

b) Constrained

Figure 7. The comparison of wall thickness change under conditions of nonrestraint and constraint

\section{Technological Experiment Research}

This experiment is carried out on the $1000 \mathrm{KN}$ universal material tester, adopting the dial indicator to measure the slide stroke, namely, decrement of the ring testing piece. Other conditions are the same as that of the simulation. Fig. 8 shows the experimental results of the compression deformability when the rolling reduction is $6 \mathrm{~mm}$ and the aluminum alloy ring is under restriction.

According to the requirements, Figure $8 \mathrm{a}$ and Figure $8 \mathrm{~b}$ show processing ring testing piece with required size and restriction to make assembly. The experimental result after compression deformability is shown in Figure 8c. It can be seen that the middle part of the inner testing piece presents invaginated trend after compression. Due to the effect of restriction, the two ends of the external ring present the change of evagination trend while the middle area basically does not change. Therefore, if the middle part of the outside is imposed with partial restriction, the internal and external surface of the ring will show the shape of evagination and drum. The ends of ring result in the trend of branch liquidity, while the material of the middle part only shows radial inflow due to the restriction. 


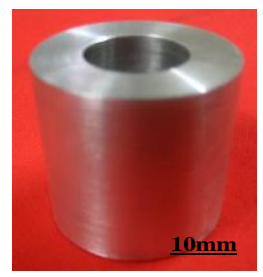

a) Original blank

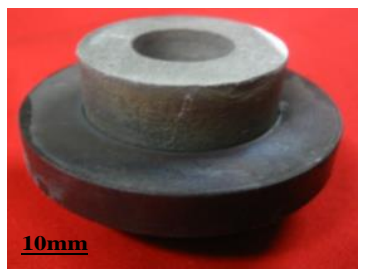

b) Local restrictions

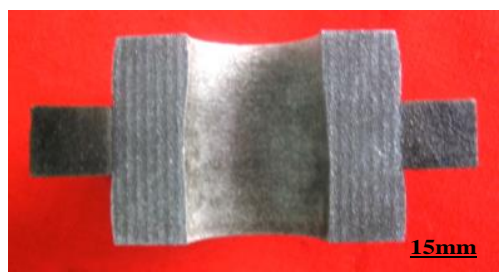

c) After compressive deformation

Figure 8. The experimental result under constraint conditions after ring compressive deformation

\section{Conclusion}

1) The research result indicates that, compared to the unrestraint situation, after restriction is imposed on the middle part of the ring outside, the area that is difficult to be deformed will be transferred to the middle part of the outside from the ends. And the branch surface lies in the two ends of the ring outside, so that it enables the formed ring outline to present pulleyshaped feature.

2) The research result which is done when the rolling reduction of the protruding model is $8 \mathrm{~mm}$ and after the compression deformability presents that, without restriction, the external diameter shows increasing trend, and the maximum thickness increasing point of the side wall lies in the middle part of the height direction, the increment is $24.6 \%$, with the restriction, the maximum thickness increasing point lies closely to the two ends, and compared to the former situation, its increment increases to twice time.

3) Through the technological experiment research, after the ring which is imposed with restriction suffers thermo-compression, the deformability of the two ends is obviously greater than that of the middle part, and the internal outline presents the shape trend of evagination and drum. Therefore, it is necessary to control the rolling reduction reasonably based on meeting the perform requirements.

\section{Acknowledgements}

This paper was financially supported by the Fundamental Research Funds for the Central Universities (DL12C B05), the Natural Science Foundation of China (31170517 and 31070500). The authors would like to take this opportunity to express their sincere appreciation.

\section{References}

[1] D. P. Dennies and M. A. Fischer, J. Fail. Anal. Prev., vol. 1, no. 4, (2004).

[2] F. Kang, Z. D. Zhao, C. K. Hu, et al., Special Casting Nonferrous Alloys., vol. 5, no. 29, (2009), (In Chinese).

[3] F. Meiners, S. Röhr and R. S. Jurgensen. Adv. Tech. Plasticity, vol. 5, (2002).

[4] F. Li, G. N. Chu and X. J. Liu, China J. Nonferrous Met., vol. 11, no. 19, (2009).

[5] G. Liu, L. L. Wang, S. J. Yuan, et al., Trans. Nonferrous Met. Soc. China, vol. 4, no. 16, (2006).

[6] G. Liu, Z. Q. Liu, L. L. Wang, et al., J. Harbin Inst. Tech., vol. 1, no. 38, (2006).

[7] J. Zhou, F. L. Wang, M. H. Wang, et al., Int. J. Adv. Manuf. Tech., vol. 1-4, no. 5, (2011).

[8] D. Q. Tan, W. X. Li, Y. D. Xiao, et al., J. Cent. South Univ. Tech., vol. 3, no. 37, (2006).

[9] A. G. Chernikov, Combust. Explo. Shock+, vol. 4, no. 12, (2007).

[10] F. Li, Y. C. Xu, S. H. Li, et al., JOM, vol. 11, no. 63, (2011).

[11] H. Zhang, D. S. Peng, L. B. Yang, et al., J. Cent. South Univ. Tech., vol. 1, no. 8, (2001). 